\title{
ASSEMBLY AND ELECTRICAL TESTS OF THE FIRST FULL-SIZE FORWARD MODULE FOR THE ATLAS ITK STRIP DETECTOR
}

\section{The ATLAS ITk Strip Detector}

- All-silicon detector with around 70 million read-out strips.

- Modular design with modules assembled in larger structures: staves and petals.

- Four barrel layers and six end-cap discs per side.

- Integrated cooling and electronics.

- Modules are made from one silicon sensor and one or more hybrid circuits.

- Read-out chips glued onto the hybrids and wire bonded for electrical connections.

- Forward region uses "Stereo Annulus" shaped sensors and modules.

- Different number of strips leads to different number of read-out chips.

- Changing strip pitch and length for each segment.

- Here, we focus on Ring $\mathbf{0}$.

Hybrid Building

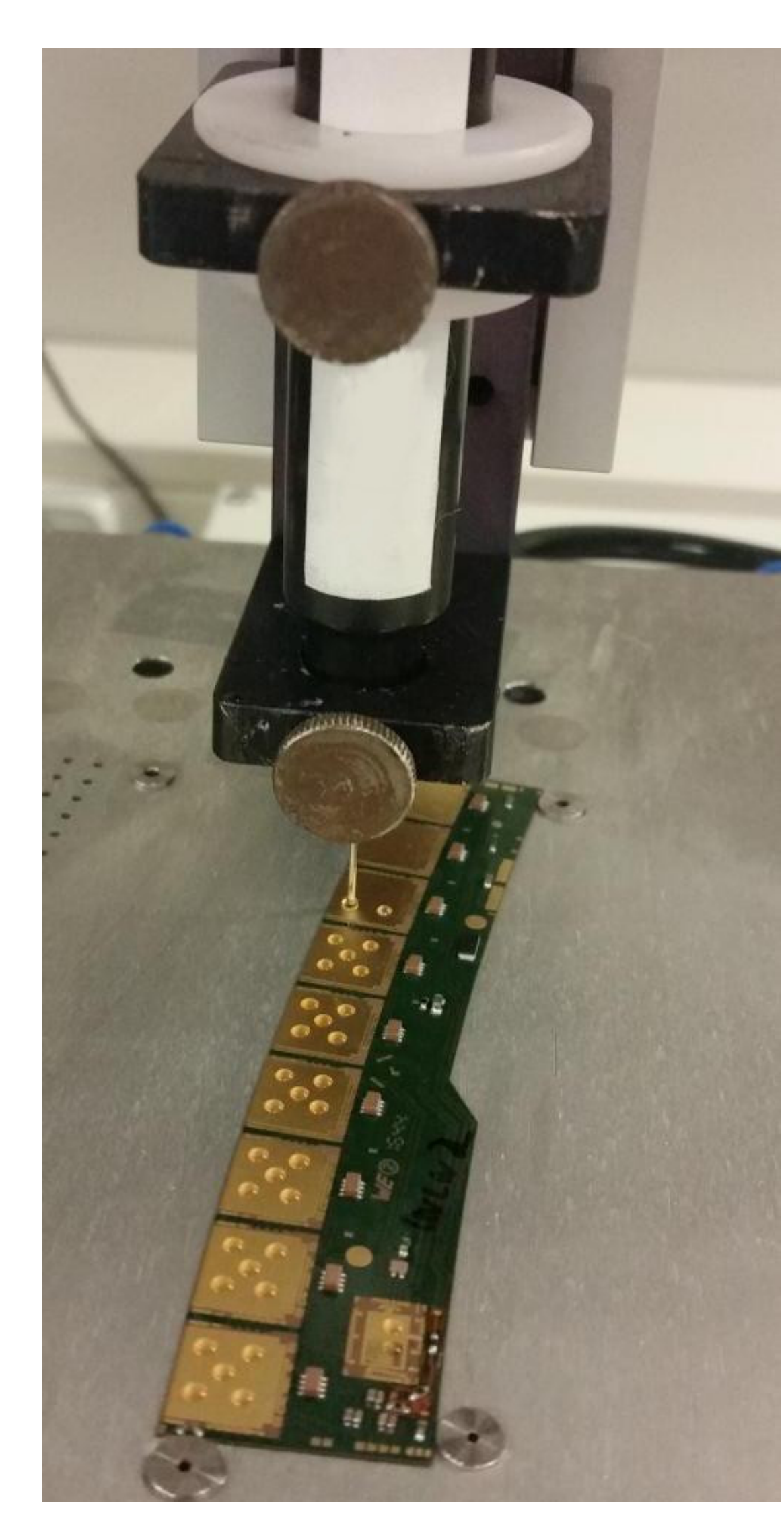

- UV-cured glue for hybrid-ASIC attachment.

- Glue dispensing robot.

- Special tooling for precise positioning and glue height control.

- Wire-bonding.

- Electrical testing.

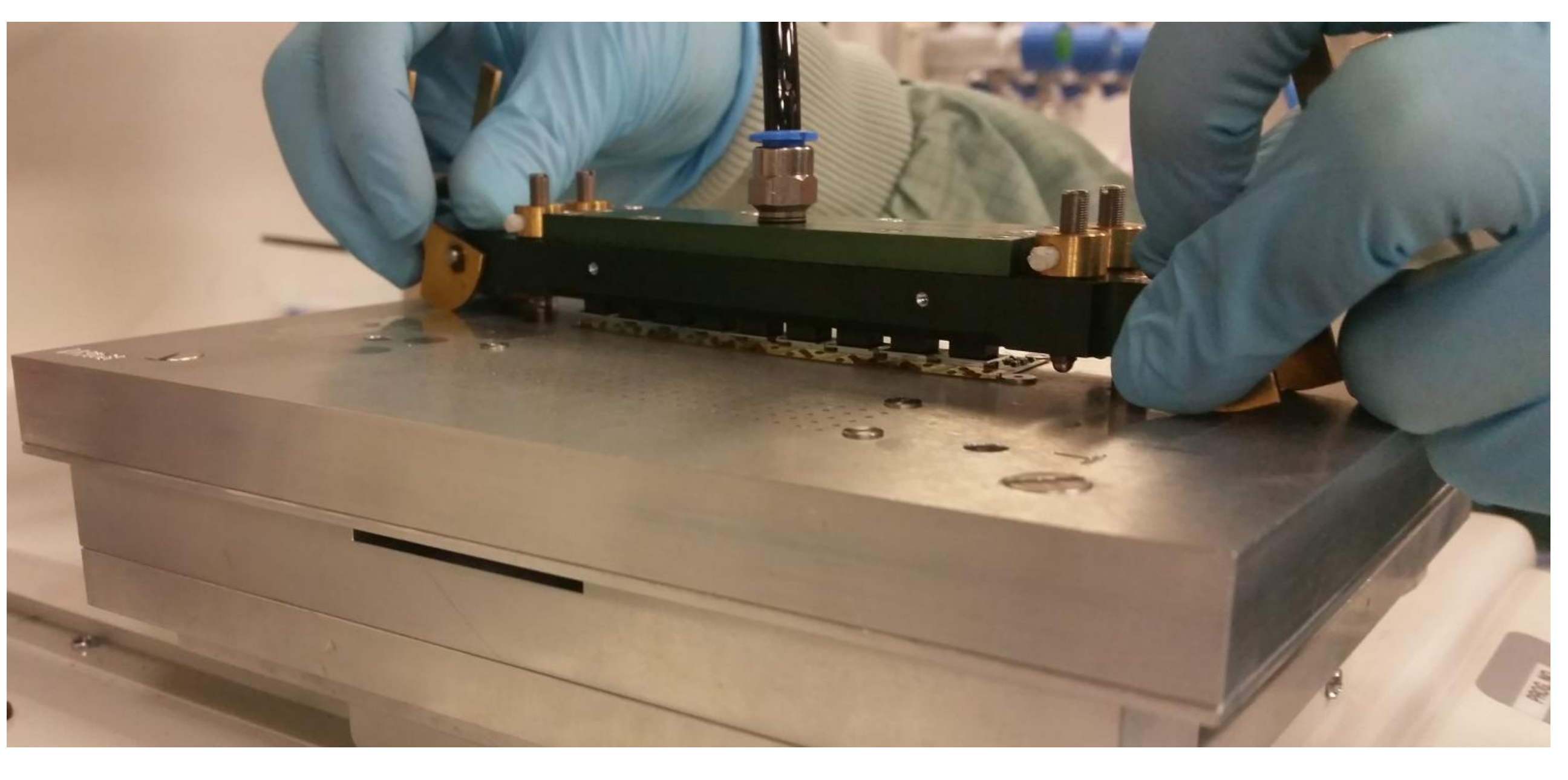

Module Building

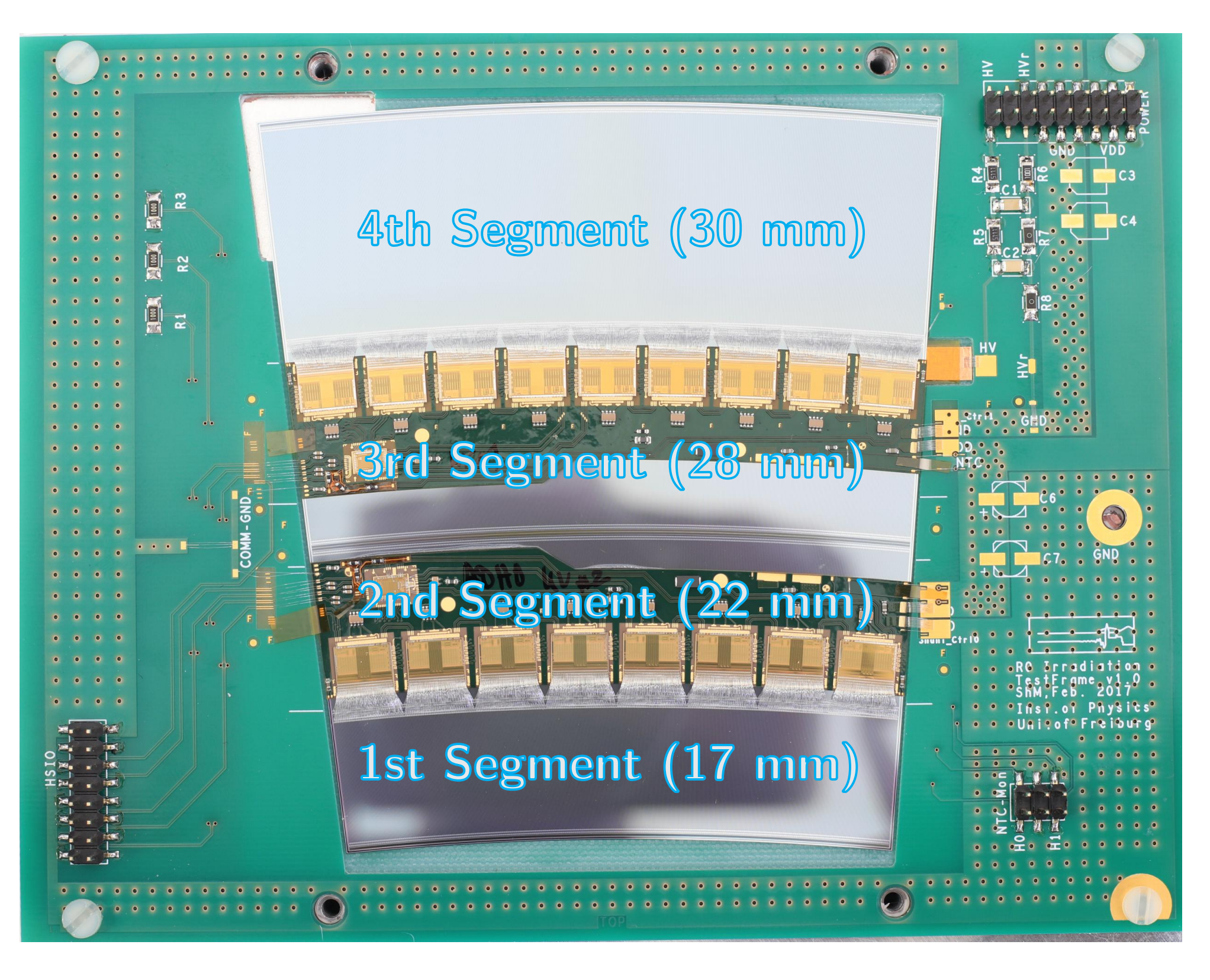

- Sensor held on precisely machined tool with vacuum.

- Epoxy glue cured for 10 hours. - Hybrid glued on sensor active area

- Electrical connections: four row wire-bonding of front-end channels.

Noise increases with strip length and decreases with hybrid-sensor glue thickness.

\section{Final Module Assembly}

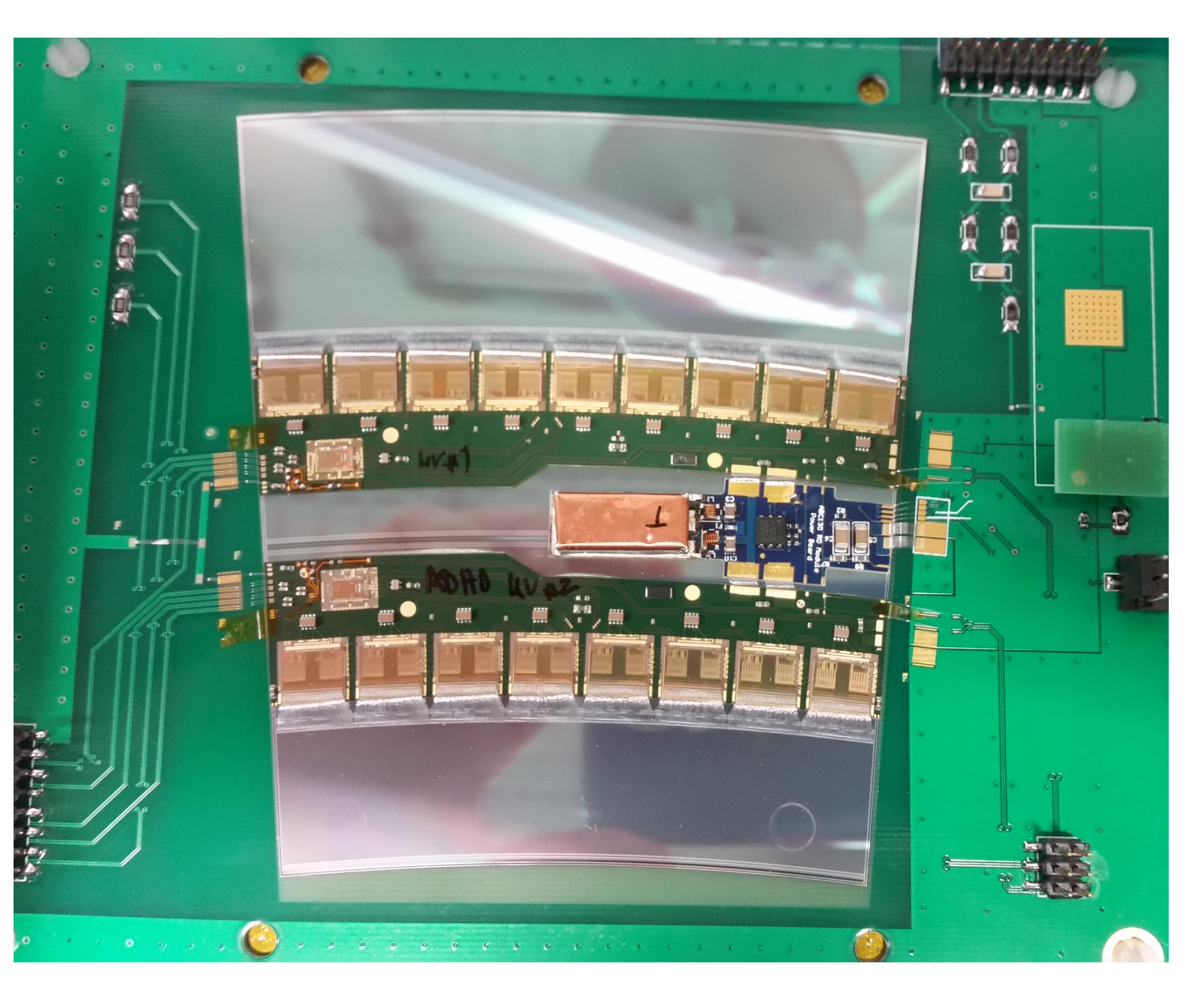

\section{- Addition of power board for} DC-DC conversion

- Buck converter using a coil for energy storage.

- Glued on top of the sensor active area with Epoxy glue.

- Wire-bonded to both hybrids and to test frame.

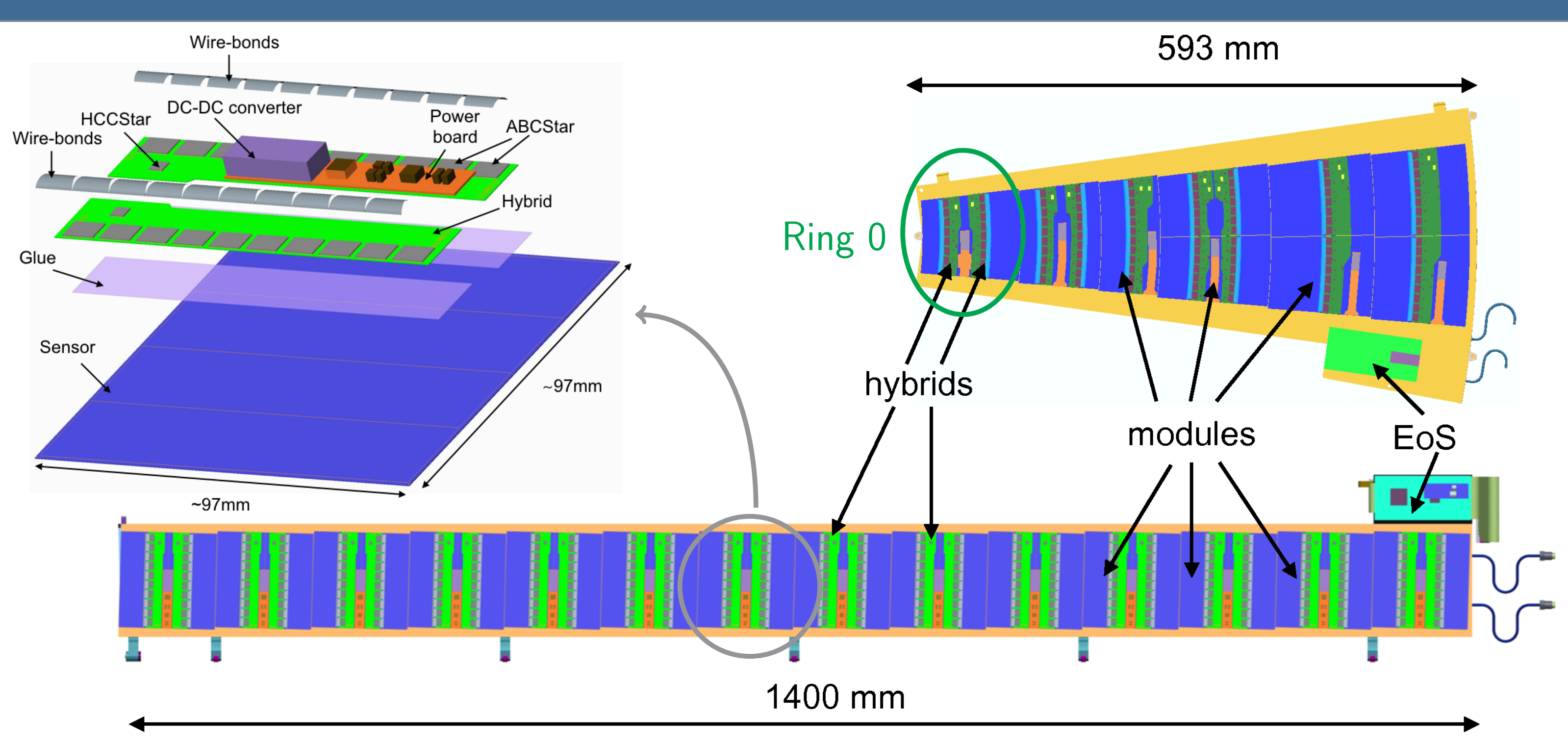

Sensor Testing

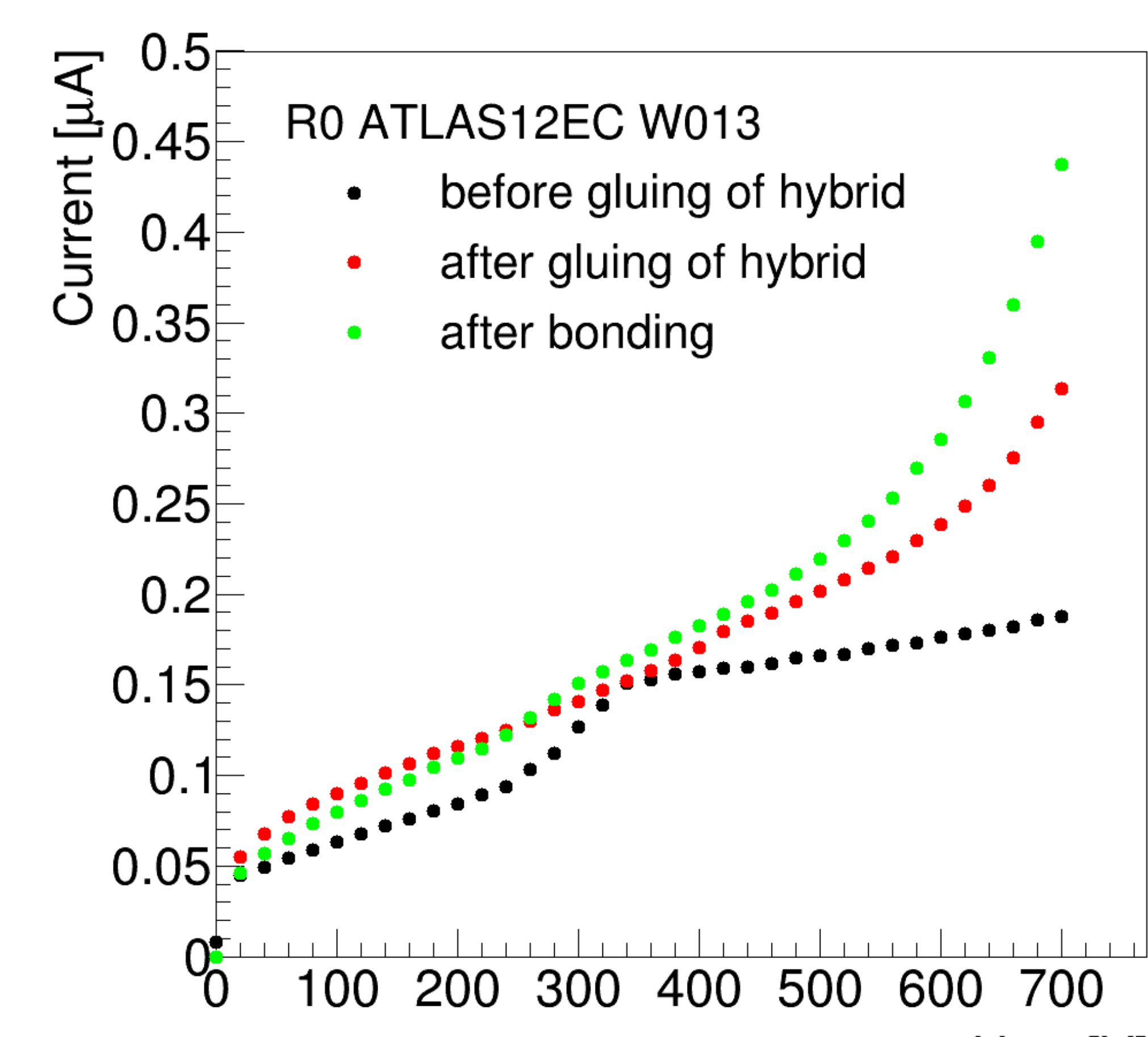

- Bare sensor measured on probe station: I/V and C/V curves.

- $\mathrm{C} / \mathrm{V}$ used to determine full depletion voltage: $\approx 300 \mathrm{~V}$.

- I/V of bare sensor, after gluing hybrids and after wire-bonding.

- Used to determine whether there is damage to the sensor.

- Slightly worsening of the I/V after gluing and wire-bonding.

- Signs of potential mechanical stress on the sensor.

- Sensitivity to humidity is another source of increased current.

\section{Initial Electrical Tests}

Characterisation by: Input

Noise (Equivalent Noise

Charge, ENC) and Noise

Occupancy.

- Tests: Response Curve

and Noise Occupancy Scan

- With changing sensor bias voltage.

- Noise behaves as expected with bias and strip length.

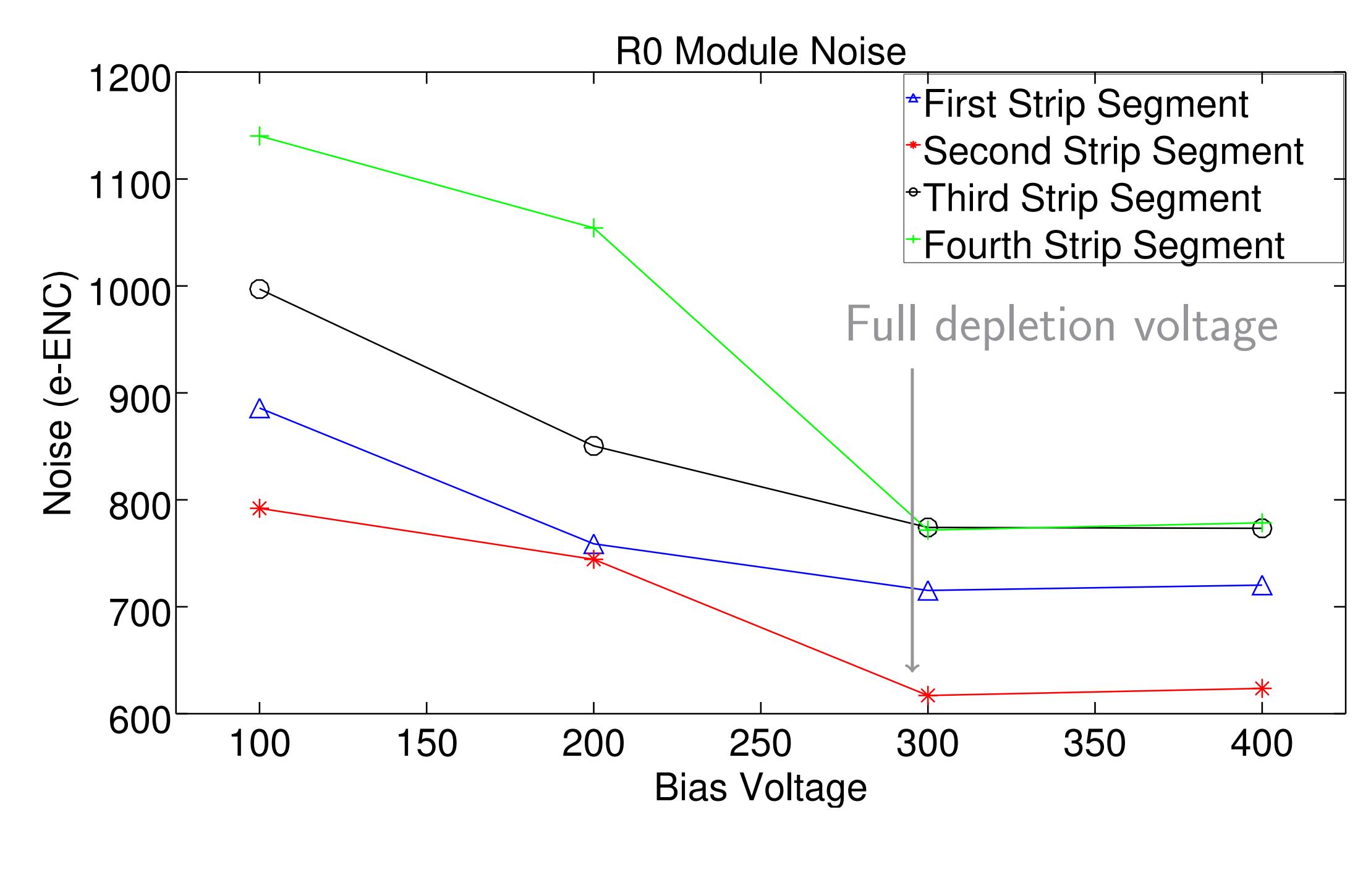

\section{Electrical Tests With Power Board}

- Slight noise increase in areas underneath the power board.

- Small B-field leakage measured next to the power board.

- We expect some noise reduction by improving the shielding.

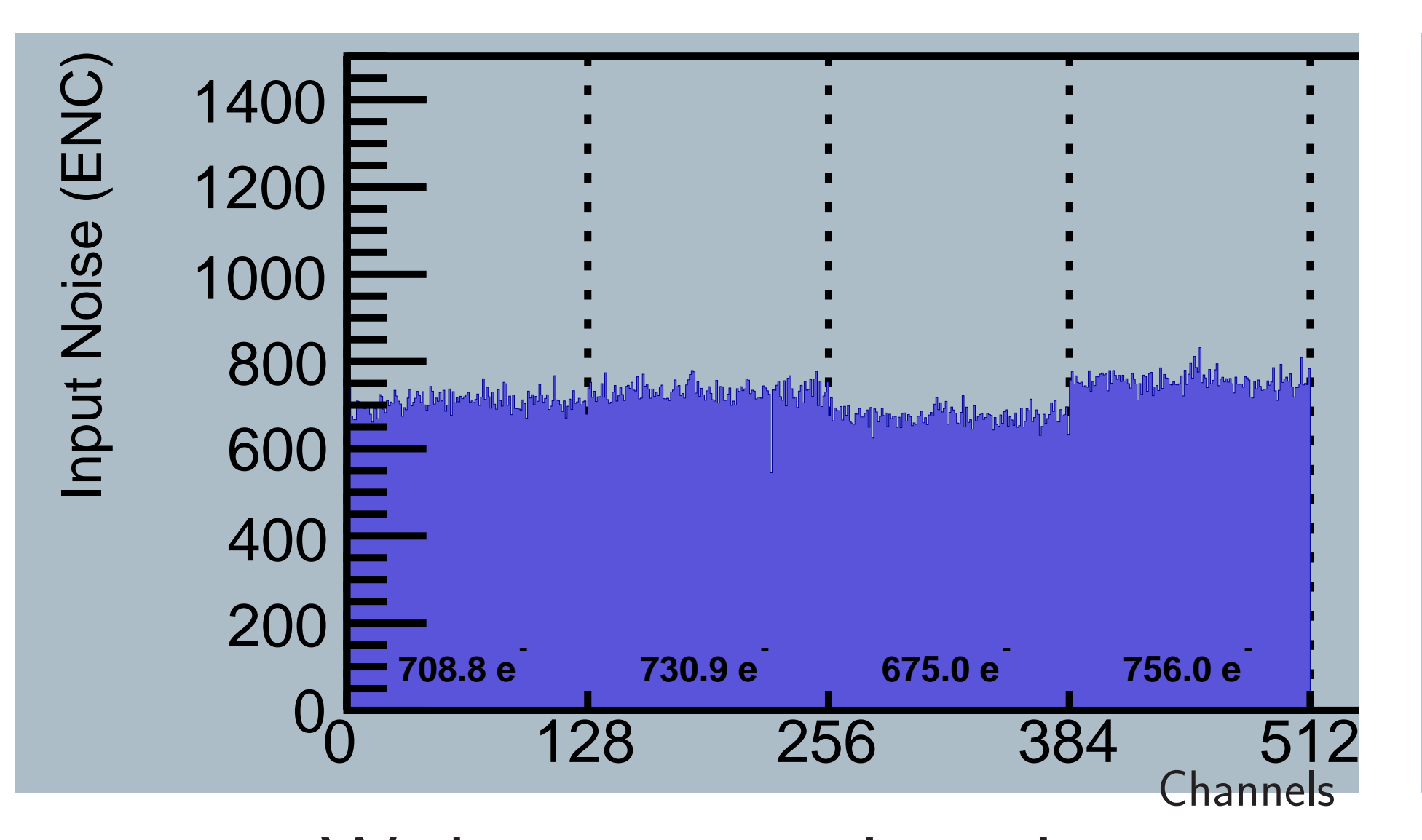

Without power board

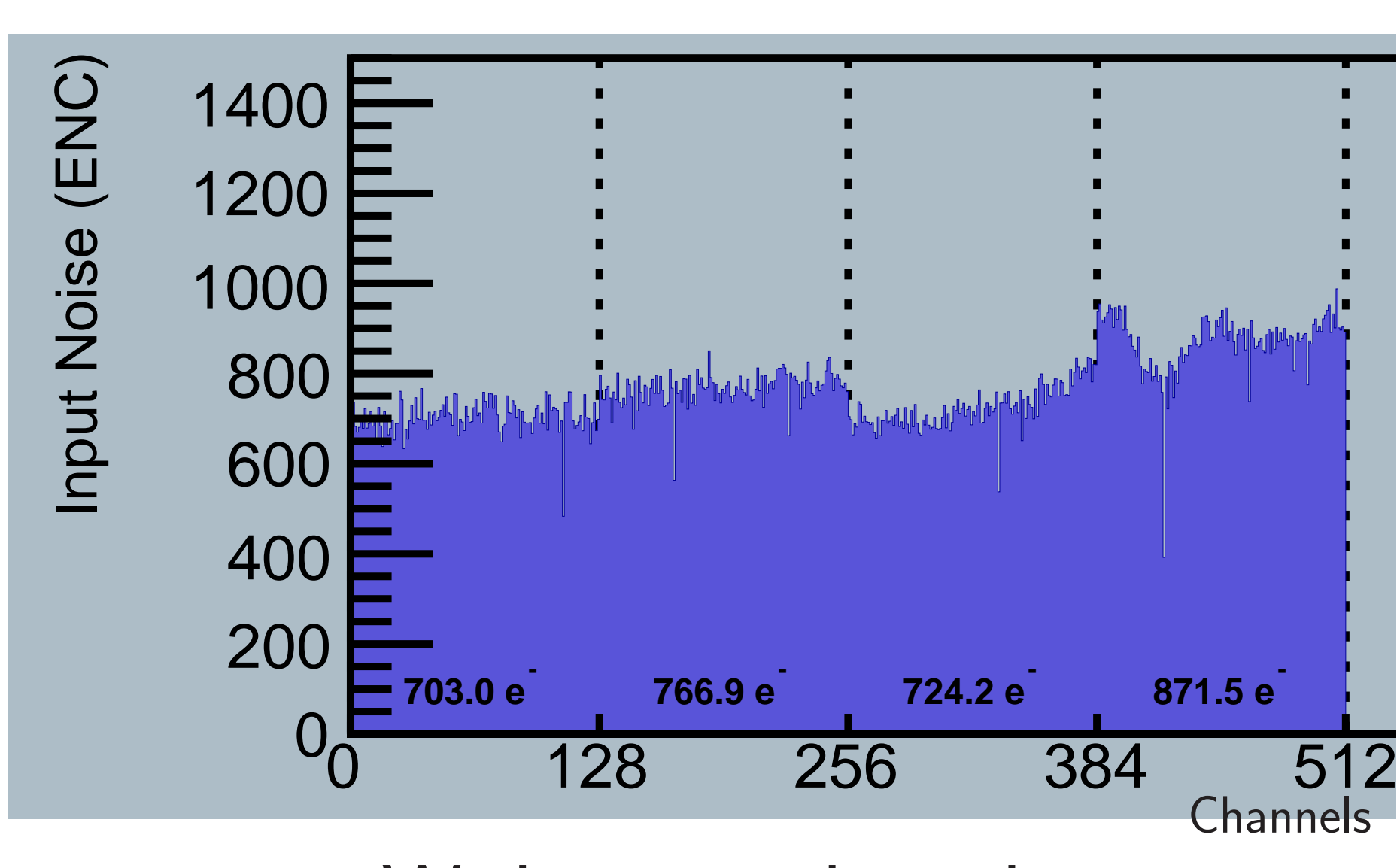

With power board

\section{Conclusions}

- The electrical tests performed so far on the first prototype end-cap sensors show good performance.

- Module building procedure is under control: precision mounting of chips, gluing to sensor and wire-bonding.

- The module noise performance is consistent with the sensor characteristics.

- Addition of a power board on top of the sensor introduces some extra noise which we expect to be able to control by improving the shielding. 